\title{
Do the current intestinal transplant criteria predict mortality in patients on home parenteral nutrition? A longitudinal assessment in a single centre
}

\author{
A. Murugananthan ${ }^{1}$, P. Paskaran ${ }^{1}$, D. A. J. Lloyd ${ }^{1}$, S. J. Middleton ${ }^{2}$ and S. M. Gabe ${ }^{1}$ \\ ${ }^{1}$ Lennard-Jones Intestinal Failure Unit, St Mark's Hospital, Harrow, UK and ${ }^{2}$ Addenbrookes Hospital, Cambridge, UK
}

Intestinal transplantation is viewed as an increasingly viable alternative to home parenteral nutrition (HPN) for patients with irreversible intestinal failure (IF). The 5-year survival of intestinal transplantation (ITx) is quoted as $37-50 \%{ }^{(1)}$. Previous work shows that 5-year survival on HPN is $73 \%^{(2)}$. It has previously been shown that $37 \%$ of patients on HPN at this institution meet the criteria for referral for transplantation $^{(3)}$. Despite this finding, only $16 \%$ of those meeting criteria for ITx in this cohort were referred for consideration of transplantation. Follow-up data are presented on the patient cohort receiving HPN from this institution in 2005. The aims were to assess: (1) the number of patients receiving HPN at St Mark's Hospital meeting criteria for ITx; (2) the number of patients referred for ITx; (3) survival rates in this patient cohort.

All patients receiving HPN on 1 January 2005 were assessed at yearly intervals for 3 years according to the Medicare ITx criteria ${ }^{(1)}$. Eligibility for referral for ITx was based on fulfilment of one or more of the following criteria over each 12-month period: advanced parenteral nutrition-associated liver disease (PNALD); thrombosis of two or more major veins; frequent central venous catheter sepsis (two or more episodes in the previous 12 months, fungaemia or septic shock); frequent severe dehydration; patient request or quality of $\operatorname{life}^{(2)}$.

In total 123 patients were reviewed in 2005, 122 in 2006 and 118 in 2007, the fall representing either discontinuation of HPN or death. The underlying aetiologies resulting in IF for these patients were Crohn's disease (31\%), vascular (27\%), pseudo-obstruction (16\%), surgical complications (13\%) and others (13\%).

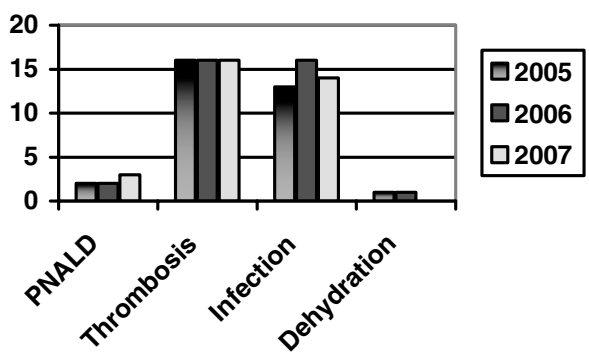

\begin{tabular}{|c|c|c|c|c|c|c|}
\hline & \multicolumn{2}{|c|}{2005} & \multicolumn{2}{|c|}{2006} & \multicolumn{2}{|c|}{2007} \\
\hline & $n$ & $\%$ & $n$ & $\%$ & $n$ & $\%$ \\
\hline Patients reviewed & 123 & & 122 & & 118 & \\
\hline Patients meeting one or more criteria & 32 & 26 & 35 & 29 & 33 & 28 \\
\hline Patients referred for consideration of ITx & 6 & 20 & 8 & 23 & 8 & 24 \\
\hline
\end{tabular}

All patients with PNALD were referred for consideration of ITx. A total of $21 \%$ of patients meeting criteria for ITx for venous thrombosis were referred whereas only $6.5 \%$ of patients meeting criteria with central venous catheter sepsis were referred over the followup period.

Eight deaths occurred at the end of the 3-year period. Two deaths occurred in patients meeting criteria for transplantation and six deaths occurred in patients who did not meet criteria.

The referral rate for consideration of ITx was considerably lower than the number of patients meeting criteria. After 3 years the mortality is similar in patients meeting ITx criteria and those not meeting criteria. At this stage the ITx criteria are not selecting patients with a poor prognosis.

1. American Gastroenterological Association (2003) Gastroenterology 124, 1111-1134.

2. Lloyd DAJ, Vega R, Bassett P et al. (2006) Aliment Pharmacol Ther 24, 1231-1240.

3. Zabron AA, Lloyd DAJ, Ralphs SJL et al. (2006) Proc Nutr Soc 66, 12A. 